\title{
A METHOD TO IMPROVE RELIABILITY OF GEARBOX FAULT DETECTION WITH ARTIFICIAL NEURAL NETWORKS
}

\author{
P.V. Srihari ${ }^{1}$, K. Govindarajulu ${ }^{2}$ and K. Ramachandra ${ }^{1}$ \\ ${ }^{1}$ Faculty of Mechanical Engineering, \\ R.V. College of Engineering, Bangalore-560 059, India, \\ Email: pvsrihari@rediffmail.com \\ ${ }^{2}$ Faculty of Mechanical Engineering, J.N.T. University, Anantapur, A.P., India.
}

\begin{abstract}
Fault diagnosis of gearboxes plays an important role in increasing the availability of machinery in condition monitoring. An effort has been made in this work to develop an artificial neural networks (ANN) based fault detection system to increase reliability. Two prominent fault conditions in gears, worn-out and broken teeth, are simulated and five feature parameters are extracted based on vibration signals which are used as input features to the ANN based fault detection system developed in MATLAB, a three layered feed forward network using a back propagation algorithm. This ANN system has been trained with 30 sets of data and tested with 10 sets of data. The learning rate and number of hidden layer neurons are varied individually and the optimal training parameters are found based on the number of epochs. Among the five different learning rates used the 0.15 is deduced to be optimal one and at that learning rate the number of hidden layer neurons of 9 was the optimal one out of the three values considered. Then keeping the training parameters fixed, the number of hidden layers is varied by comparing the performance of the networks and results show the two and three hidden layers have the best detection accuracy.
\end{abstract}

Keywords: Gearbox fault diagnosis, Vibration signal, artificial neural networks, Reliability.

\section{INTRODUCTION}

Gear boxes are widely used in typical industries like steel, power, oil, airplane, helicopter and automobile and they are complex machinery where many rotating elements are in action with one another. Depending on the criticality of the machine, condition monitoring and fault diagnosis becomes important which in other terms is also related to the reliability of condition monitoring. Among the many condition monitoring methods, vibration monitoring plays an important role in increasing maintainability. Fault detection and diagnosis consists of feature extraction and decision making where the first step is the mapping from the signal space to the feature space and the second step is classifying the features into different categories, also called fault detection. The reliability of the diagnosis depends on the expertise in relating the vibration features to the faults, where sometimes signatures are contaminated by noise, contradicting symptoms, and limitations on the ability of human beings when multiple features are applied. An effort has been made to develop an ANN based system (Yegnanarayana, 1998) for gear fault diagnosis and detection that increases the reliability of diagnosis. 
An efficient maintenance schedule can be planned if accurate information about the machine condition is known, and to improve the reliability of diagnostics either combining two technologies or data fusion and intelligent systems (Farrar and Duffey, 1999) has been found useful. Vibration based diagnosis is mostly employed because of its ease of measurement and the accuracy of detection of the original signal with phase modulation and amplitude modulation (Wang et al., 2001). The detection and classification accuracy of an ANN based bearing diagnosis system is 97.4-100 \% (Jack and Nandi, 2002). The key factor for a proper fault classifying system is to select the best suitable input values, which would be the base classifying system (Bartelmus et al., 2003). The detection accuracy of support vector machines is better than ANN without genetic algorithm (GA). With GA both are comparable (Samantha et al., 2003). The training parameters play a vital role in deciding the operational efficiency of the neural network, such as the number of neurons in the hidden layer, the learning rate, and the number of epochs (Amarnath et al., 2005).

\section{PREPARATION OF INPUT VALUES TO ANN}

A gearbox with 16/14 helical teeth as gear and pinion is to be studied here. The required vibration signature is collected from the signal generator using an accelerometer of PCB peizotronics make with a sensitivity of $100 \mathrm{mV} / \mathrm{g}$. Different sets of data were collected when the gearbox was operating in two fault conditions viz., worn out case and broken tooth case. A total of 30 sets of data were collected for each operating condition. The signals were sampled at $12 \mathrm{kHz}$. The accelerometer outputs are inherently amplified as the accelerometer employed is of ICP type. These are fed directly into the USB powered LDS Dactron, Photon II, 4 channel analyzer that acts as the data acquisition system. The response of the system is averaged over 10 measurements with a Hanning window. The vibration signature is preprocessed in order to obtain the required features of mean, root mean square (rms), variance, skewness and kurtosis. These features act as the input parameters to the artificial neural network which is developed using MATLAB neural network toolbox. A three layer feed forward network is employed and trained using the back propagation algorithm.

The training parameters play a vital role in deciding the operational efficiency of the neural network. Training of a neural network involves the selection of some key parameters such as the number of epochs, the number of neurons in the hidden layer, and the learning rate. After training, the network is validated using test data. Among the 30 sets of data collected, 20 are used for training the network and 10 are used as the test data. The learning rate is varied at $0.05,0.1,0.15,0.2$ and 0.25 while keeping the number of hidden layer neurons constant at 9 and in turn based on the number of epochs an optimal design is decided as shown in Figure 1. The detection performance obtained for various cases is given in Table 1. From the results shown in Table 1, the learning rate of 0.15 is deduced to be the optimal one considering the number of epochs and also the accuracy of the outputs. In the next step, the learning rate is kept constant at 0.15 and the number of hidden neurons is varied from 5 to 15 . Table 2 shows the outputs of the network while varying the number of hidden layer neurons. It can be clearly seen that 9 hidden layer neurons is the most optimal one. In this way, the optimum training parameters have been deduced. Then the network was trained and tested for these values. Figure 2 shows the variation in the mean square error with respect to the number of epochs while reaching the target value of 0.0001 during training of the network. Closeness of the target vectors and outputs obtained for our network are shown in Table 
3. A five dimensional vector consisting of mean, kurtosis, rms, skewness and variance represents each data set. The detection accuracy of the trained neural network is $100 \%$ as shown in Table 4.

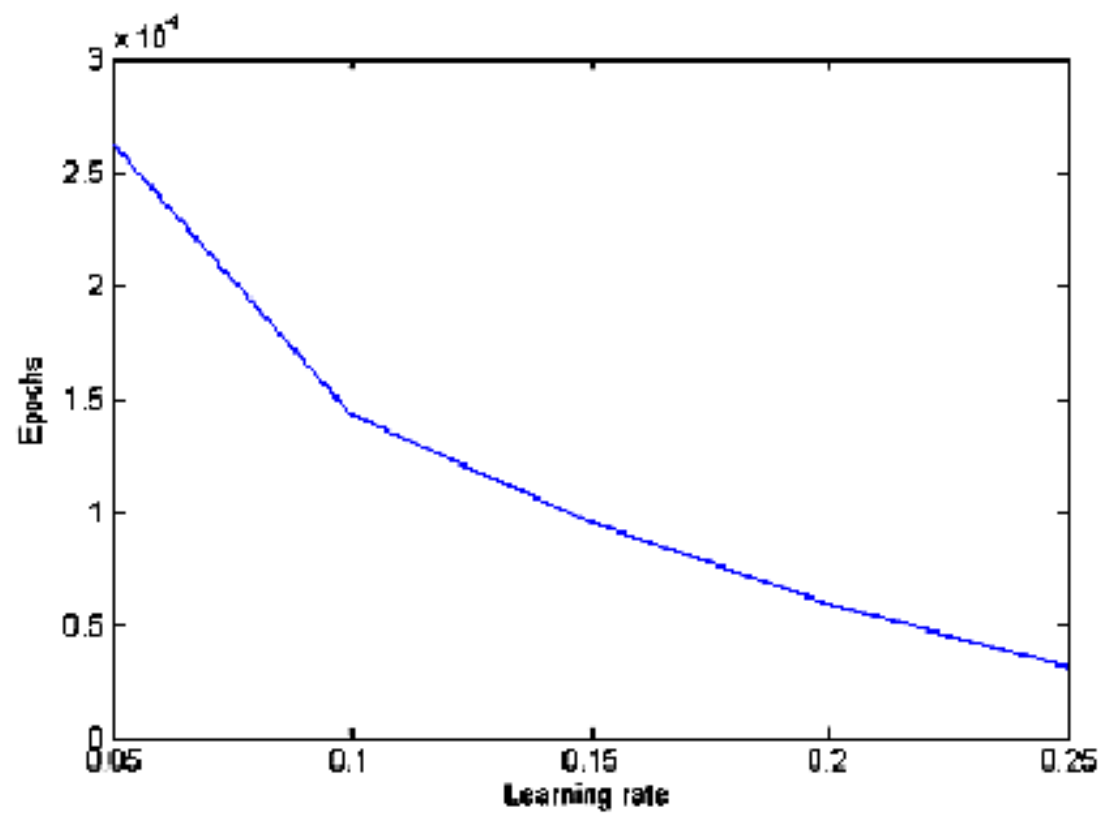

Figure 1. Number of epochs vs learning rate

Table 1. Number of the epochs vs accuracy of the outputs

\begin{tabular}{ccccc}
\hline S. No & $\begin{array}{c}\text { Learning } \\
\text { rate }\end{array}$ & Epochs & $\begin{array}{c}\text { Case 1 } \\
{[\mathbf{1 , 0}]}\end{array}$ & $\begin{array}{c}\text { Case 2 } \\
{[\mathbf{0 , 1}]}\end{array}$ \\
\hline 1 & 0.05 & 26914 & {$[0.94,0.03]$} & {$[0.04,0.93]$} \\
2 & 0.10 & 14263 & {$[0.93,0.02]$} & {$[0.04,0.94]$} \\
3 & 0.15 & 9500 & {$[0.98,0.01]$} & {$[0,0.99]$} \\
4 & 0.20 & 5938 & {$[0.95,0.05]$} & {$[0.05,0.96]$} \\
5 & 0.25 & 3126 & {$[0.94,0.03]$} & {$[0.04,0.95]$} \\
\hline
\end{tabular}

Table 2. Outputs with hidden layer neurons

\begin{tabular}{ccccc}
\hline $\begin{array}{c}\text { S. } \\
\text { No }\end{array}$ & $\begin{array}{c}\text { Hidden layer } \\
\text { neurons }\end{array}$ & Epochs & $\begin{array}{c}\text { Worn out case } \\
\text { output }\end{array}$ & $\begin{array}{c}\text { Cracked case } \\
\text { output }\end{array}$ \\
\hline 1 & 5 & 12,138 & {$[0.9941,0.0111]$} & {$[0.0113,0.9968]$} \\
2 & 9 & 9500 & {$[0.9894,0.0082]$} & {$[0.0002,0.9958]$} \\
3 & 15 & 6583 & {$[0.9919,0.0088]$} & {$[0.0104,0.9889]$} \\
\hline
\end{tabular}




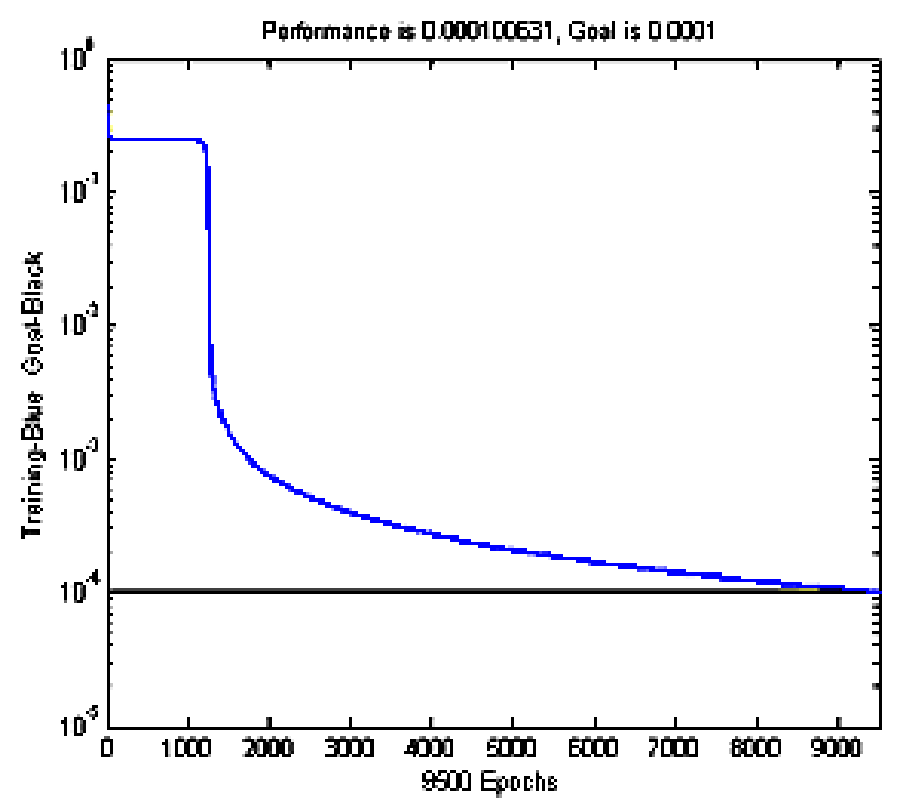

Figure 2. Training the network

Table 3. Outputs for learning rate of 0.15

\begin{tabular}{ccc}
\hline Case & Output obtained & Target \\
\hline Worn out gear & {$[0.998,0.008]$} & {$\left[\begin{array}{ll}1 & 0\end{array}\right]$} \\
Cracked gear & {$[0.0002,0.998]$} & {$[01]$} \\
\hline
\end{tabular}

Table 4. Detection accuracy

\begin{tabular}{ccc}
\hline Case & $\begin{array}{c}\text { No. of inputs } \\
\text { identified }\end{array}$ & $\begin{array}{c}\text { Detection } \\
\text { accuracy }\end{array}$ \\
\hline Worn out gear & $10 / 10$ & $100 \%$ \\
Cracked gear & $10 / 10$ & $100 \%$ \\
\hline
\end{tabular}

\section{ANN BASED FAULT DETECTION}

The vibration signature is collected from the signal generator using a B\&K 4332 Accelerometer with a sensitivity of $104 \mathrm{mV} / \mathrm{g}$ mounted at the bearing housing of the 16 teeth pinion, meshing frequencies are calculated to be $320 \mathrm{~Hz}$ and multiples. Different sets of data are collected when the gearbox is operating healthy at $20 \%, 40 \%, 80 \%$ tooth worn out conditions and completely the broken tooth condition. A total of 30 sets of data are collected for each operating condition. The signals are truncated to $3 \mathrm{kHz}$ using a low pass filter and sampled at $8 \mathrm{kHz}$. The accelerometer outputs are conditioned using a B\&K 2626 charge amplifier. The frequency response of the system averaged over 10 measurements with a Hanning window was obtained using an Agilent 3570A analyzer.

The vibration signature is preprocessed in order to obtain the required features of mean, root mean square (rms), standard deviation, skew ness and kurtosis. These features act as the input parameters to the artificial neural network. The neural network is developed using the MATLAB neural network toolbox. A feed forward network is 
employed and trained using the back propagation algorithm. Among the 30 sets of data collected, 20 were used for training the network and 10 sets were used as the test data. Keeping the training parameters constant and varying the number of hidden layers of the artificial neural network, the performance of the networks was compared. The training parameters considered for this investigation are as follows:

- Number of hidden layer neurons $=15$

- Performance function for the first hidden layer $=$ Tan - Sigmoidal

- Performance function for the second hidden layer $=\log -$ Sigmoidal

- Performance function for the output layer $=\log -$ Sigmoidal

- Learning rate $=0.15$

- $\quad$ Training goal $=0.01$

When the inputs are applied to the network, if the output pattern appears [1 0$]$, then the network indicates that the inputs belong to the worn-out case; and if the output pattern appears as [0 1], then the network indicates that the inputs belong to the broken tooth case as listed in Table 5. For the experimental investigation, the output pattern pertaining to five different faults for each error case are listed in Table 6.

Table 5. Output pattern for fault classification

\begin{tabular}{cc}
\hline Case & Pattern \\
\hline Worn out & {$\left[\begin{array}{ll}1 & 0\end{array}\right]$} \\
Broken tooth & {$\left[\begin{array}{ll}0 & 1\end{array}\right]$} \\
\hline
\end{tabular}

Table 6. Output pattern for fault detection

\begin{tabular}{|c|c|}
\hline Case of operation & Output pattern \\
\hline Healthy gear & {$\left[\begin{array}{lllll}1 & 0 & 0 & 0 & 0\end{array}\right]$} \\
\hline $20 \%$ worn out gear & {$\left[\begin{array}{lllll}0 & 1 & 0 & 0 & 0\end{array}\right]$} \\
\hline $40 \%$ worn out gear & {$\left[\begin{array}{lllll}0 & 0 & 1 & 0 & 0\end{array}\right]$} \\
\hline $80 \%$ worn out gear & {$\left[\begin{array}{lllll}0 & 0 & 0 & 1 & 0\end{array}\right]$} \\
\hline 1 Broken tooth & {$\left[\begin{array}{lllll}0 & 0 & 0 & 0 & 1\end{array}\right]$} \\
\hline
\end{tabular}

Based on the output pattern, the network diagnoses the class to which the given particular set of inputs belongs, and thus gives the output stating the corresponding class. If the output pattern generated by the input is of the order $\left[\begin{array}{lllll}0 & 1 & 0 & 0 & 0\end{array}\right]$ then the network immediately displays that the given set of inputs belong to the case of $20 \%$ wear thus indicating that there is $20 \%$ wear on one of the teeth. In this way ANN will be effectively able to identify and at the same time easily communicate the error to the user. Thus it can be deduced that with the selection of the optimum features and parameters and proper training procedures, this neural network can classify gear faults with the desired accuracy.

\section{RESULTS AND DISCUSSION}

The training of the network with one hidden layer is shown in Figure 3 which needed 34,500 epochs in order to reach the required error goal. A neural network with two 
hidden layers in training took 24,200 epochs to reach the error goal depicted in Figure 4. The network with three hidden layers shown in Figure 5 needs 17,249 epochs to reach the error goal. A network with four hidden layers is shown in Figure 6 which needed 22,333 epochs. The detection capability of all the networks is analysed. The networks have been tested for all 50 test inputs. The performance of all networks is compared in Table 7. The variation in the number of epochs with each case is depicted in Figure 7. From the results obtained in Table 5, it is clearly seen that the networks with two or three hidden layers have a better detection accuracy than the other cases. But the amount of memory occupied by a three hidden layer network is much greater than the two hidden layers network. As the accuracy capability of both layers is the same, the two hidden layers network is the optimum, accounting for the amount of memory consumed.

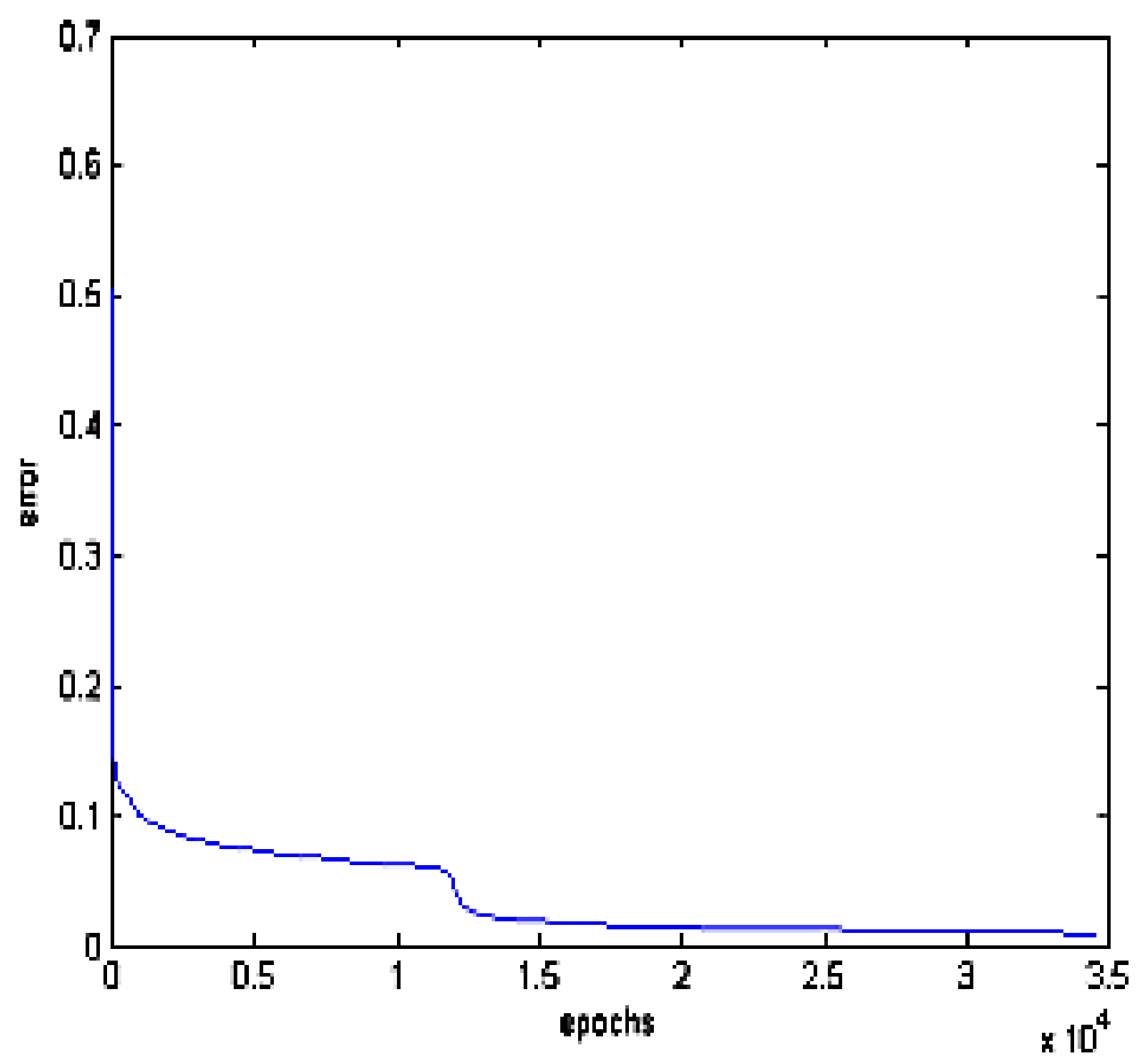

Figure 3. One hidden layer network 


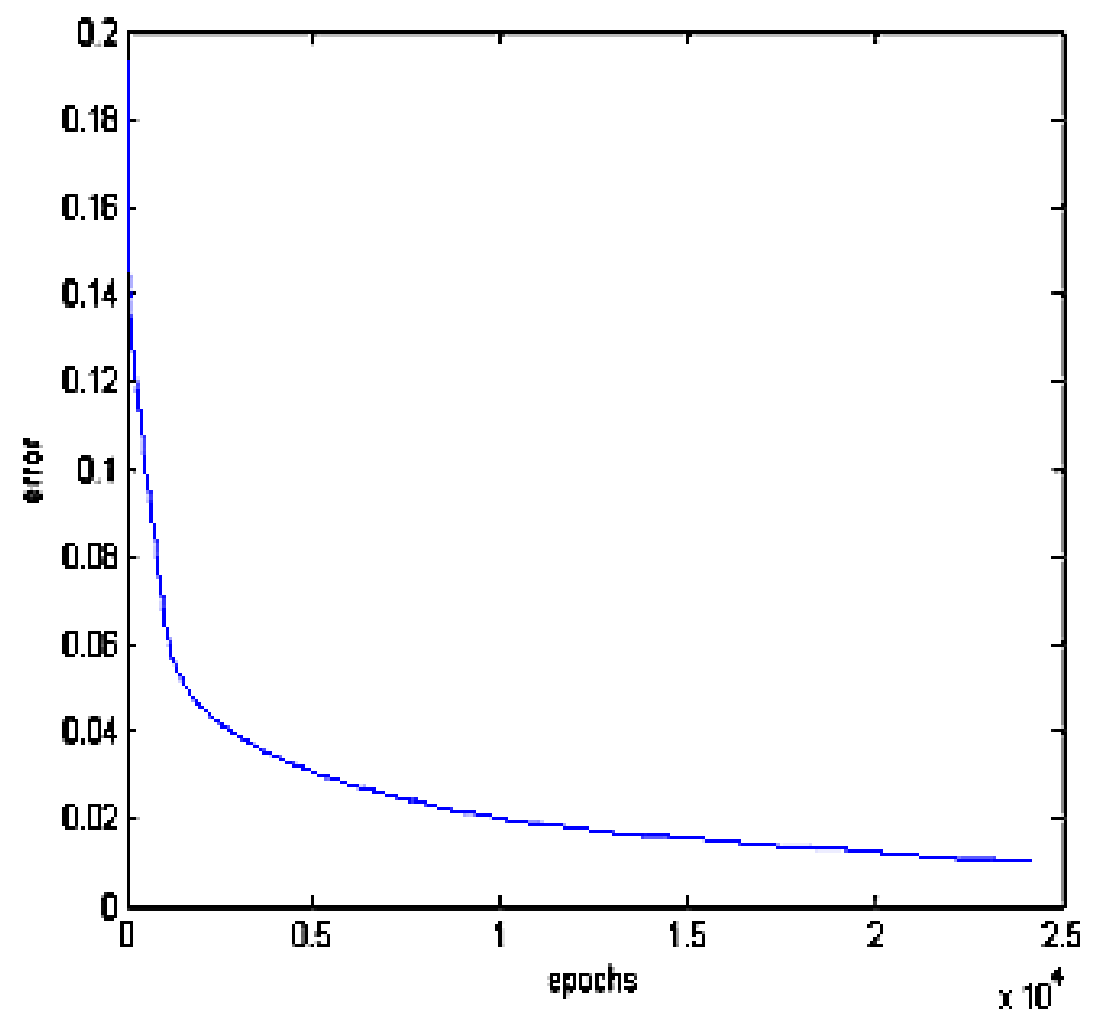

Figure 4. Two hidden layer network

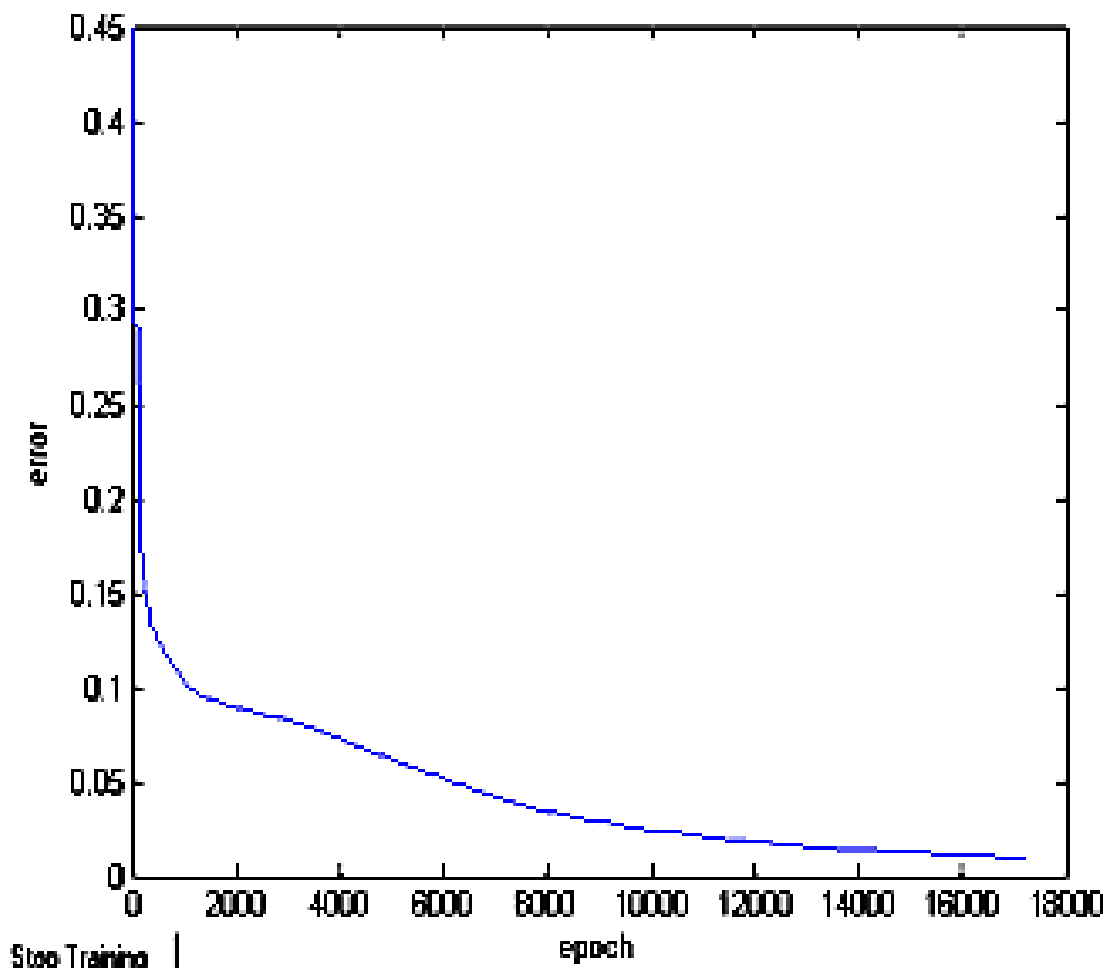

Figure 5. Three hidden layer network 


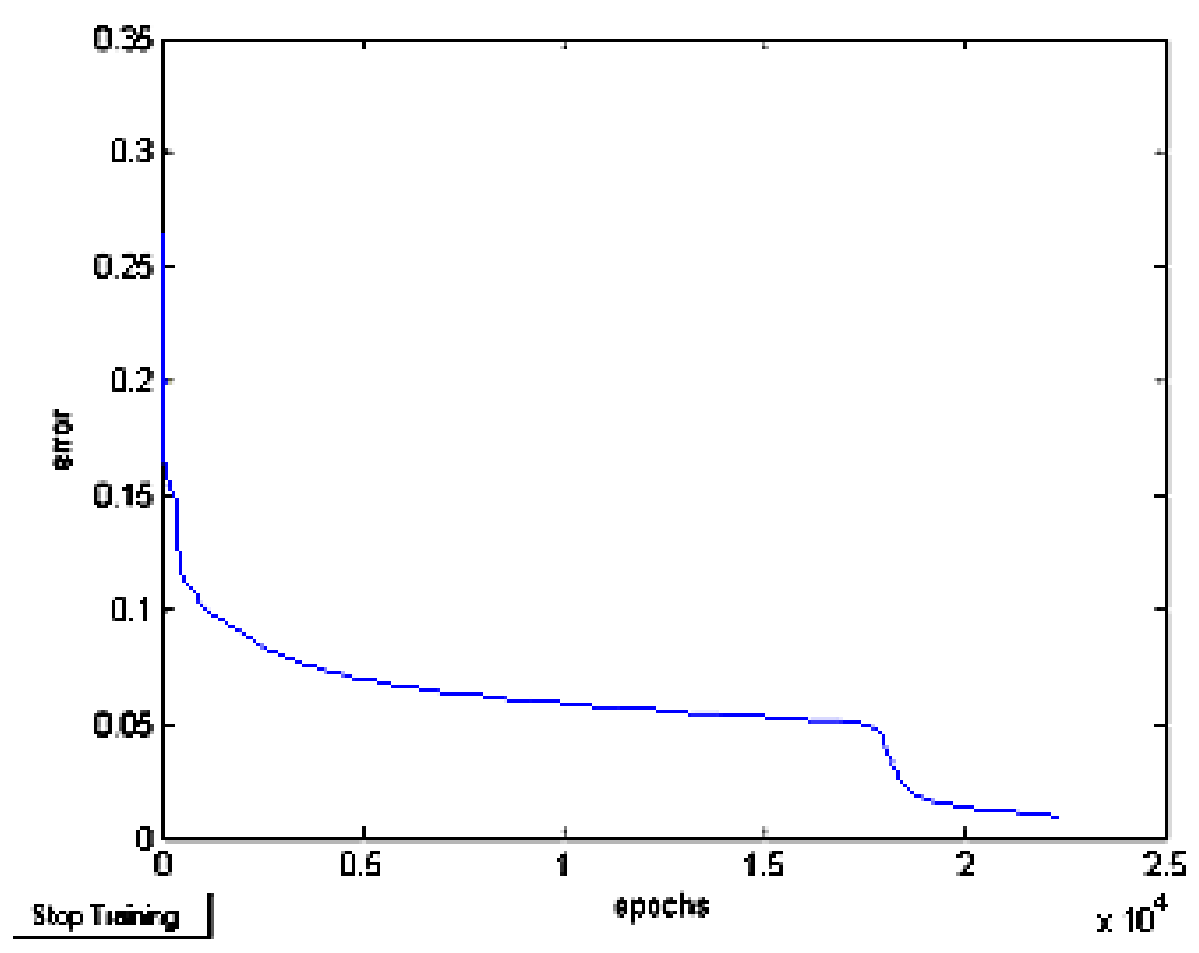

Figure 6. Four hidden layer network

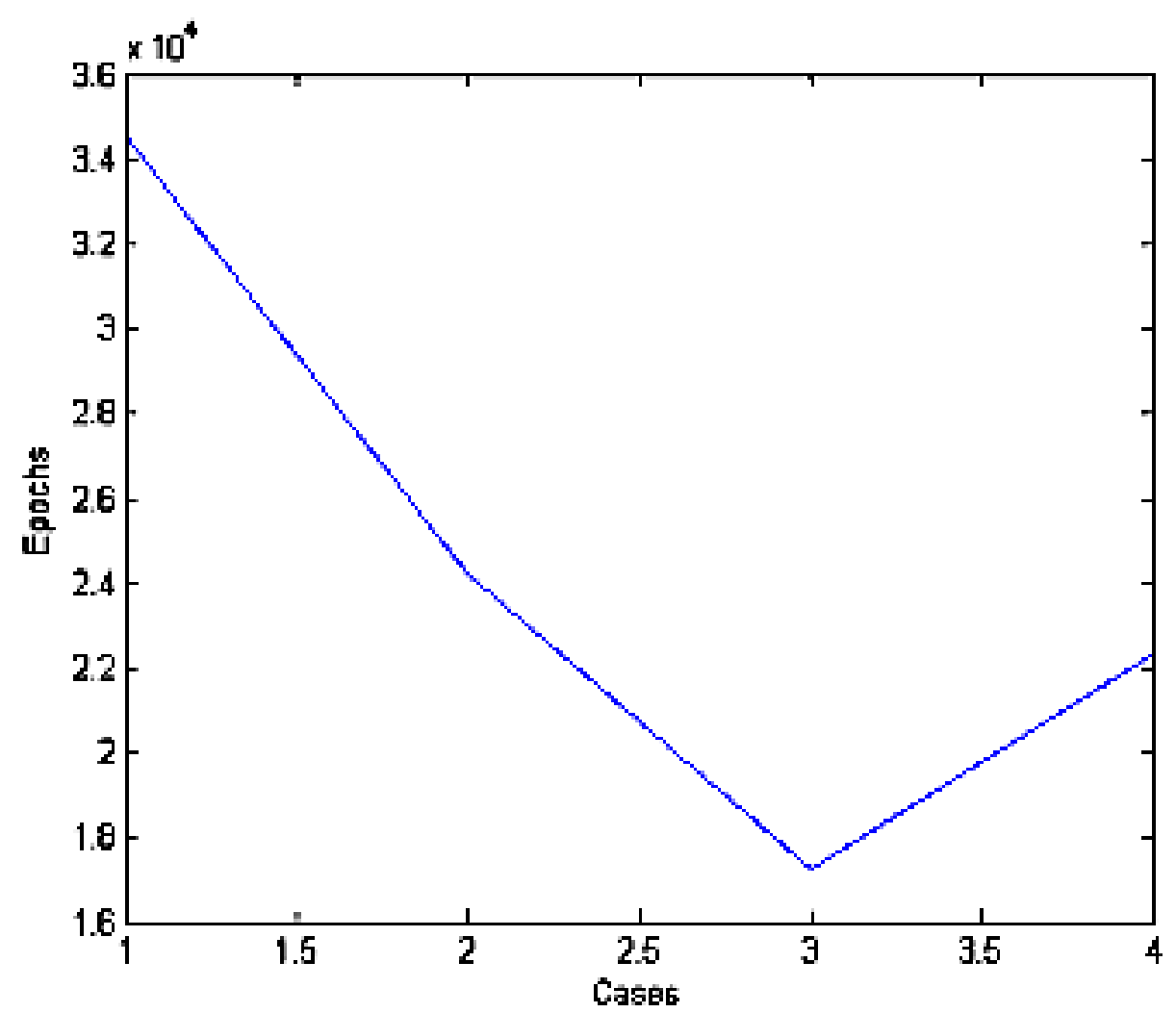

Figure 7. Number of hidden layers vs number of epochs 
Table 7 Performance of the networks

\begin{tabular}{cccc}
\hline S. No & Network type & $\begin{array}{c}\text { Number of inputs } \\
\text { properly identified }\end{array}$ & $\begin{array}{c}\text { Classification } \\
\text { accuracy }\end{array}$ \\
\hline 1 & One hidden layer & $42 / 50$ & $84 \%$ \\
2 & Two hidden layers & $47 / 50$ & $94 \%$ \\
3 & Three hidden layers & $47 / 50$ & $94 \%$ \\
4 & Four hidden layers & $43 / 50$ & $86 \%$ \\
\hline
\end{tabular}

\section{CONCLUSIONS}

An ANN based fault detection is developed using the time domain features extracted from their vibration signal for two prominent operating conditions: worn-out gear case and cracked tooth case. The design parameters of the neural network are carefully selected as they play a vital role in the construction of the model. Among the five different learning rates considered between 0.05 to 0.25 , the 0.15 is deduced to be optimal one and at that learning rate 9 hidden layer neurons is the optimal one out of three values of consideration between 5 to 15. Detection accuracy of the investigations shows that the number of hidden layers plays a vital role in determining the optimum neural network. Considering the constraint of memory consumption, ANN consisting of 2 hidden layers is chosen to be the optimum one despite having the same detection accuracy as that of the ANN having three hidden neurons based on the memory consumed. Detection accuracy is $100 \%$ as demonstrated in this work using multi layer feed forward network can be effectively used in improving the reliability of the fault detection process through appropriate measurements.

\section{ACKNOWLEDGEMENTS}

The authors would like to thank J.N.T.U.A. University, Anantapur, A.P. and R.V. College of Engineering, Bangalore for encouraging our research and Dr. Ing. B.V.A. Rao, retired Professor, IITM, Chennai, for his guidance.

\section{REFERENCES}

Amarnath, M., Swarnamani, S., Sujatha, C. (2005) Classification of Gear Wear Using Artificial Neural Networks. Twelfth International Congress on Sound and Vibration (ICSV12), p. 477.

Bartelmus, W., Zimroz, R., Batra, H. (2003) Identification of Optimized Diagnostic Features of Multi-Stage Gearbox Condition. 18th International Conference on Systems Engineering, pp. 434-439.

Farrar, C.R. and Duffey, T.A. (1999) Vibration Based Damage Detection in Rotating Machinery. Proc. of DAMAS, pp. 224-235.

Jack, L.B. and Nandi, A.K. (2002) Fault Diagnosis Using Support Vector Machines and Artificial Neural Networks Augmented by Genetic Algorithm. Mechanical Systems and Signal Processing, 16(2-3): 373-390.

Samantha, B., Al Balushi, K.R. and Al-Araimi, S.A. (2003) Artificial Neural Network Based Fault Diagnosis of Rolling Element Bearings Using Time-Domain Features. Engineering Applications of Artificial Intelligence, 16(7-8): 657-665. 
Samantha, B. (2003) Gear Fault Detection Using Artificial Neural Networks and Support Vector Machines With Genetic Algorithms. Mechanical Systems and Signal Processing, 18(3): 625-644.

Wang, W.Q., Ismail, F. and Golnaraghi, M.F. (2001) Assessment of Gear Damage Monitoring Techniques Using Vibration Measurements. Mechanical Systems and Signal Processing, 15(5): 905-922.

Yegnanarayana, B. (1998) Artificial Neural Networks. New Delhi: Prentice-Hall. 